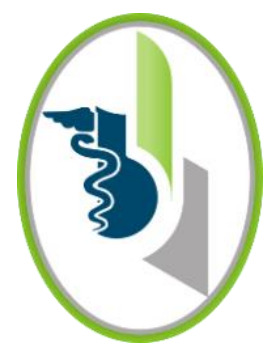

Para citaciones: Corrales, D., Arias, J. (2020). Los probióticos y su uso en el tratamiento de enfermedades. Revista Ciencias Biomédicas, 9(1), 54-66.

Recibido: 8 de septiembre de 2019 Aprobado: 2 de diciembre de 2019

Autor de correspondencia: Daniela Corrales Benedetti danielacobe24@gmail.com

Editor: Inés Benedetti. Universidad de Cartagena-Colombia.

Copyright: (C) 2020. Corrales, D., Arias, J. Este es un artículo de acceso abierto, distribuido bajo los términos de la licencia https://creativecommons.org/licenses/bync-sa/4.0/ la cual permite el uso sin restricciones, distribución y reproducción en cualquier medio, siempre y cuando el original, el autor y la fuente sean acreditados.

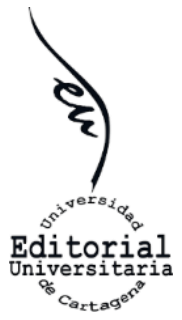

\section{Los probióticos y su uso en el tratamiento de enfermedades}

\author{
Probiotics and their use in the treatment of diseases.
}

\author{
Daniela Corrales Benedetti ${ }^{1}$, Janeth Arias Palacios ${ }^{2}$ id
}

${ }^{1}$ Facultad de Ciencias, Programa de Microbiología, Pontificia Universidad Javeriana. Bogotá, Colombia.
${ }^{2}$ Facultad de Ciencias, Programa de Microbiología, Pontificia Universidad Javeriana. Bogotá, Colombia.

\section{RESUMEN}

Introducción: Los probióticos son microorganismos vivos que confieren beneficios a la salud del huésped, cuando se consumen en cantidades adecuadas, proporcionando una acción protectora contra patógenos y beneficios nutricionales. Su uso está asociado principalmente a alimentos funcionales o suplementos dietarios que contribuyen a un mejoramiento de la salud principalmente en humanos. Sin embargo, en los últimos años se han venido estudiando nuevos usos de estos, como son la prevención y alivio de ciertas enfermedades principalmente gastrointestinales. Así mismo los probióticos se han convertido en una alternativa para el tratamiento de enfermedades infecciosas, para mitigar el efecto negativo que los antibióticos pueden generar sobre el microbioma de los pacientes.

Objetivo: El objetivo de esta revisión bibliográfica fue identificar y compilar estudios e investigaciones en las cuales se evidencie el papel que juegan los probióticos en la solución de dichos problemas.

Métodos: Se realizó una revisión sistemática de artículos, de los cuales se seleccionaron aquellos que permitieron dar respuesta a los objetivos planteados.

Resultados: Se logro identificar diferentes estudios donde se evidencio el beneficio del uso de los probióticos para el tratamiento de diferentes enfermedades.

Conclusiones: Los probióticos son una novedosa alternativa en el tratamiento contra patógenos y en la prevención de algunas enfermedades en humanos. Ya que estos contribuyen en la reducción del agente causal de la enfermedad, reducción de los síntomas, inducción de la actividad inmune o disminución en el riesgo de contraer la infección.

Palabras Clave: probióticos; Bacillus spp; Sacharomyces spp; Lactobacillus spp; Faecalibacterium prausnitzii; Bifidobacterium.

\section{ABSTRACT}

Introduction: probiotics are live microorganisms that confer benefits to the health of the host, when consumed in adequate quantities, providing a protective action against pathogens and nutritional benefits. Its use is mainly associated with functional foods or dietary supplements that contribute to an improvement of health, mainly in humans. However, in recent years new uses of these have been studied, such as the prevention and alleviation of certain diseases mainly gastrointestinal. Likewise, probiotics have become an alternative for the treatment of infectious diseases, to 
mitigate the negative effect that antibiotics can generate on the microbiome of patients.

Objective: the aim of this review was to identify and compile the latest studies and research in which the role of probiotics in the solution of such problems is evidenced.

Methods: A systematic review of articles was carried out, of which those that allowed us to respond to the proposed objectives were selected.

Results: It was possible to identify different studies where the benefit of the use of probiotics for the treatment of different diseases was evidenced.

Conclusion: Probiotics are a novel alternative in the treatment against pathogens and in the prevention of some diseases in humans. Cause these contribute to the reduction of the causative agent of the disease, reduction of symptoms, induction of immune activity or reduction of the risk of contracting the infection.

Keywords: probiotics; Bacillus spp; Lactobacillus spp; Faecalibacterium prausnitzii; Bifidobacterium.

\section{INTRODUCCIÓN}

La definición que se conoce internacionalmente de probióticos es la de microorganismos vivos que cuando se administran en cantidades adecuadas, confieren un beneficio para la salud del huésped. La mayoría de los probióticos que se han estudiado son bacterias, entre las cuales se encuentran principalmente las bacterias ácidos lácticas (BAL), también se han estudiado algunos mohos y levaduras, los cuales han mostrado tener un potencial probiótico (1). Estos son considerados organismos beneficiosos ya que su uso puede proporcionar ciertos efectos positivos al huésped como, mantener el equilibrio del microbioma intestinal, inhibir el crecimiento de bacterias patógenas, sintetizar y mejorar la biodisponibilidad de nutrientes, promover una buena digestión, reducir el efecto de los alérgenos, reducir el colesterol, estimular el sistema inmune, aliviar la intolerancia a la lactosa y aumentar la resistencia a la infección (2).

El uso de probióticos está asociado principalmente a alimentos funcionales o suplementos dietarios que contribuyen a un mejoramiento de la salud en humanos. Sin embargo, en los últimos años se han venido estudiando nuevos usos de estos como, la prevención y alivio de ciertas enfermedades principalmente gastrointestinales, para la cuales los tratamientos existentes traen consigo efectos secundarios en los pacientes o simplemente no existían métodos efectivos para manejarlas. Así mismo, los probióticos se han convertido en una alternativa para el tratamiento de enfermedades infecciosas, para mitigar el efecto negativo que los antibióticos pueden generar sobre el microbioma de los pacientes.

Como bien se sabe el sector salud actualmente enfrenta una serie de problemas. Uno de estos es la falta de tratamiento de algunas enfermedades y los efectos secundarios de los tratamientos existentes, especialmente de padecimientos gastrointestinales. Por otro lado, está el uso de antibióticos, que, aunque son el principal tratamiento cuando se habla de enfermedades infecciosas, estos no solo atacan a los microorganismos patógenos, sino que también causan efectos negativos sobre los microorganismos presentes en el microbioma de los pacientes. A demás, puede llevar a la aparición de resistencia por parte de microorganismos. Por lo que el uso de probióticos se ha convertido en un método alternativo, para el tratamiento de algunas enfermedades. El objetivo de esta revisión bibliográfica fue identificar y compilar estudios e investigaciones en las cuales se evidencie el papel que juegan los probióticos en la solución de dichos 
problemas, además se determinaron los mecanismos de acción de cepas probióticas frente a patógenos y los principales patógenos sobre los cuales actúan los probióticos utilizados en la salud humana.

\section{MÉTODOS}

Se realizó una revisión sistemática de artículos de las bases de datos Science Direct, SpingerLink, Medline, Science Journals, ProQuest y Scopus, se seleccionaron aquellos que permitieron dar respuesta a los objetivos planteados. El intervalo de tiempo que se tuvo en cuenta fue a partir del año 2010 hasta el año 2018. Se utilizaron diferentes palabras claves en la búsqueda de artículos, empleando las siguientes palabras: probióticos, enfermedades, gastro- intestinales, Bacillus spp , Sacharomyces spp, Lactobacillus spp, Faecalibacterium prausnitzii, Akkermansia muciniphila y Bifidobacterium.

Se desarrollaron cuatro etapas para la elaboración de la revisión. En la primera, se realizó la búsqueda e identificación de los artículos o documentos en las bases de datos, teniendo en cuenta principalmente la identificación de estudios realizados que permitieran dar respuesta a los objetivos propuestos. En la segunda, se realizó la recopilación de la información encontrada previamente para así continuar con la tercera etapa, en donde se realizó el análisis de esta. Por último, se hizo un análisis más detallado de la información, realizando una organización, evaluación y selección para así identificar y dar respuesta a la revisión propuesta.

\section{RESULTADOS}

\section{Generalidades de los probióticos}

Se puede decir que el termino probiótico surgió hace muchos años con las civilizaciones antiguas, ya que los griegos y los romanos, usaban productos lácteos fermentados para mantener la salud. Sin embargo, la investigación sobre microorganismos en los productos alimenticios fermentados y sus efectos sobre la salud humana sólo se comenzó a estudiar recientemente. Uno de los primeros registros de la actividad de estos se obtuvo en 1908, cuando el premio Nobel Élie Metchnikoff, en el Instituto
Pasteur, estableció la relación de la salud y la longevidad con la ingestión de bacterias a partir del yogurt. Metchnikoff propuso que las bacterias ayudaron a controlar las infecciones causadas por patógenos entéricos, y que además regulaban la toxemia, las cuales desempeñan papeles importantes en el envejecimiento y la mortalidad (3).

Probiótico es una palabra derivada del latín y el griego, que significa "para la vida", que fue utilizada por primera vez por Kollath en1953 (4). Lilly y Stillwell en 1965 fueron los primeros en proponer una definición de probióticos basados en sus investigaciones donde observaron como el crecimiento de protozoos in vitro fue estimulado por otros protozoos. La definición que le dieron a esto fue de sustancias secretadas por un microorganismo que estimulan el crecimiento de otro. Durante la década siguiente, el término probiótico también fue utilizado por Fujii y Cook quienes en 1973 se refirieron esta vez a productos químicos sintéticos en ratones que conferían protección contra la infección por Staphylococcus aureus (5).

En 2001 un panel de expertos encargados por la Organización de las Naciones Unidas para la Agricultura y la Alimentación y respaldado por la Organización Mundial de la Salud, proporcionó una definición clara del término probiótico la cual fue "Microorganismos vivos que cuando se administran en cantidades adecuadas otorgan un beneficio en la salud del huésped " (6).

En los últimos años han surgido otras definiciones donde se han tenido en cuenta la especificación de los mecanismos, el sitio de acción y el huésped. En 2013, la Organización Mundial de Gastroenterología publicó sus directrices globales sobre probióticos y prebióticos, y confirmó que la eficacia de los probióticos es específica de la cepa y dosis, disipando el mito de muchos de que cualquier yogurt puede considerarse un probiótico. Igualmente, en el 2014 en un panel convocado por la Asociación Científica Internacional para Probióticos y Prebióticos, se determinó el alcance y el uso apropiado del término probiótico y se recomendó que dicho término se use sólo en productos que 
contengan microorganismos vivos con un recuento viable adecuado de cepas bien definidas, con una expectativa razonable de ofrecer beneficios para el bienestar del huésped. Así mismo se definieron tres categorías de probióticos: (I) aquellos sin declaraciones de propiedades saludables (generalmente considerados seguros, no se necesita prueba de eficacia), (II) como un complemento alimenticio con un objetivo de salud específico (cepa utilizada definida, eficacia basada en la evidencia de ensayos clínicos o metaanálisis, uso para reforzar las defensas naturales o reducir los síntomas), o (III) como un fármaco probiótico (ensayos clínicos para indicación o enfermedad específica, cepa definida utilizada, justificación de riesgo-beneficio, cumpliendo con los estándares regulatorios para drogas) (7).

Cepas con actividad probiótica: la mayoría de los probióticos son bacterias, entre las cuales las BAL son el tipo más común, pero algunos mohos y levaduras también se pueden usar como probióticos. Los géneros comúnmente utilizados como probióticos incluyen, las bacterias Lactobacillus, Bifidobacterium, Lactococcus, Carnobacterium, Enterococcus, Streptococcus, Pediococcus, Propionibacterium, Leuconostoc y especies de Bacillus, levaduras como Saccharomyces, y hongos como Aspergillus (8).

Criterios de un buen probiótico: deben cumplir los siguientes criterios:

- Debe ser un organismo que sea capaz de ejercer un efecto beneficioso sobre los huéspedes.

- Debe ser no patógeno y no tóxico.

- Debe sobrevivir y metabolizarse en el entorno intestinal al resistir el bajo pH del estómago, ácidos orgánicos, ácidos biliares y enzimas presentes en los intestinos.

- Ser estable en condiciones de almacenamiento y campo (9).

Beneficios de los probióticos: los beneficios de los probióticos se pueden clasificar sobre la base de tres modos de acción. (I) Los probióticos podrían modular las defensas del huésped, incluidos tanto el sistema inmune innato como el adquirido. Es muy probable que este modo de acción sea importante para la prevención y tratamiento de enfermedades infecciosas, pero también para el tratamiento de la inflamación crónica del tracto digestivo. (II) Los probióticos también pueden tener un efecto directo sobre otros microorganismos, comensales y/o patógenos. Este efecto es importante en la prevención y el tratamiento de infecciones y en la restauración del equilibrio microbiano en el intestino. (III) Finalmente, los efectos probióticos pueden basarse en acciones que afectan a productos microbianos como toxinas, productos hospedadores, por ejemplo: sales biliares e ingredientes alimentarios. Tales acciones pueden dar como resultado la desactivación de toxinas y la desintoxicación del huésped y los componentes del alimento en el intestino (10).

\section{Mecanismos de acción}

Existen muchos mecanismos por los cuales los probióticos pueden ejercer sus efectos, logrando así la prevención $\mathrm{y}$ tratamiento de infecciones, el aumento del valor nutricional de los alimentos, mejorando la salud intestinal y confiriendo beneficios para la salud. Estos mecanismos son, la producción de sustancias antimicrobianas, inhibición de la adherencia epitelial y de la mucosa de los patógenos, competencia por nutrientes limitados, producción de ácidos, actividad antitoxina, secreción de moléculas, competencia, inhibición de la invasión y adhesión epitelial por patógenos, modulación del sistema inmune y de la actividad del microbioma intestinal (11); siendo estos dependientes de cada cepa, por lo que es vital seleccionar y analizar cada probiótico individualmente para su propósito previsto.

Actividad antimicrobiana: los mecanismos de actividad antimicrobiana de los probióticos desempeñan un papel principal en el equilibrio entre microorganismos beneficiosos y potencialmente patógenos (12). Los microorganismos probióticos aplican este mecanismo al secretar una variedad de compuestos antimicrobianos, que incluyen ácidos orgánicos, peróxido de hidrógeno, bacteriocinas y biosurfactantes, que pueden inhibir el crecimiento de 
bacterias patógenas. Se ha reportado que Lactobacillus reuteri produce el antibiótico reuterina (3- hidroxipropionaldehído), el cual es un antibiótico de amplio espectro, no solo contra bacterias Gram positivas y Gram negativas, sino también contra levaduras, hongos, protozoos y virus (13). Igualmente se ha visto que Lactobacillus spp. produce una variedad de sustancias que inhiben el crecimiento de bacterias Gram positivas y Gram negativas. Las sustancias inhibidoras incluyen bacteriocinas, peróxido de hidrógeno y ácidos orgánicos. Estos compuestos pueden reducir no solo la cantidad de células bacterianas viables, sino también afectar el metabolismo bacteriano y su producción de toxinas. Siendo las bacteriocinas un mecanismo de defensa importante contra microorganismos patógenos (14). Por ejemplo, la bacteriocina de amplio espectro de clase II Abp118 producida por la cepa UCC118 de Lactobacillus salivarius es capaz de proteger a ratones contra la infección de Listeria monocytogenes transmitida por alimentos (15).

Dicho lo anterior al liberar compuestos antimicrobianos, los probióticos pueden suprimir el crecimiento de patógenos transmitidos por los alimentos, y también pueden reducir el desarrollo de biopelículas por patógenos y defenderse contra la infección.

Producción de ácidos: en general, las bacterias probióticas del ácido láctico liberan ácidos orgánicos (principalmente ácido láctico y acético) que reducen el $\mathrm{pH}$ intestinal. Esto lleva a la reducción de colonización por patógenos, además crean condiciones más adecuadas para el microbioma residente y crean un ambiente ácido inhibidor de patógenos (16).

Estos ácidos muestran una actividad antimicrobiana más fuerte en comparación con las sales biliares sintetizadas por el organismo huésped (10).

Actividad enzimática: los probióticos también pueden emplear mecanismos enzimáticos, secretando enzimas capaces de hidrolizar toxinas bacterianas, modificar los receptores de toxinas e inhibir la enfermedad mediada por toxinas (17). Por ejemplo, Alvarez-Olmos y Oberhelman demostraron que la secreción de enzimas poliaminas permitía a Saccharomyces boulardii degradar los receptores de toxina de Clostridium difficile en el íleon de conejo y bloquear la secreción inducida por cólera en el yeyuno de rata (17). Así mismo, se demostró que Bifidobacterium breve Yakult y Bifidobacterium pseudocatenulatum DSM20439 inhibieron la expresión de la toxina shiga en cepas de E. coli (STEC) O157: H7, tanto en experimentos In vitro como en ratones (18).

Mecanismos metabólicos: las cepas probióticas también pueden mostrar mecanismos metabólicos, en los que crean un ecosistema anaeróbico favorable en el intestino para el microbioma residente mediante la desintoxicación de moléculas inhibidoras y compuestos que eliminan el oxígeno, como aminas o nitratos. Estas actividades metabólicas y la capacidad de supervivencia en todo el intestino dependen principalmente de las cepas probióticas utilizadas (19). Las cepas probióticas también pueden secretar exopolisacáridos (EPS) que pueden inhibir la formación de biopelículas por patógenos (20). Los EPS son un gran grupo de biopolímeros que se producen durante el proceso metabólico de los microorganismos. Además, se ha demostrado que los EPS son importantes para las BAL debido a su importante rol en la resistencia al estrés, la adhesión, la colonización y las interacciones entre el huésped y la bacteria (21).

Uno de los EPS utilizado a nivel mundial es el secretado por Lactobacillus plantarum. En estudios realizados se encontró que el EPS de L. plantarum posee actividad antibacteriana y antiinflamatoria, además de tener capacidad anti-adhesión contra patógenos presenten en las células epiteliales en el intestino humano, especialmente de Escherichia coli O157:H7. Igualmente, el EPS exhibió una fuerte inhibición contra la formación de biopelículas por bacterias patógenas, incluyendo Pseudomonas aeruginosa, E. coli O157: H7, Salmonella Typhimurium y Staphylococcus aureus (21). 
Competencia por recursos limitados: un ejemplo importante de una sustancia limitante es el hierro, debido a que, para casi todas las bacterias, el hierro es un elemento esencial, menos para lactobacilos. Estos no necesitan hierro en su hábitat natural, lo cual podría ser una ventaja crucial en la competencia con otros microorganismos que dependen del hierro. Sin embargo, L. acidophilus y L. delbrueckii son capaces de unir el hidróxido férrico en la superficie de sus células, lo que lo hace inaccesible para los microorganismos patógenos (10).

Adhesión y colonización: algunos candidatos probióticos también pueden utilizar mecanismos de prevención de la colonización, en los que se inhiben competitivamente la adhesión de microorganismos patógenos a las superficies de las células huésped (22). Para este mecanismo, las proteínas particulares que están presentes en la superficie de los agentes probióticos son importantes. Como, las proteínas de la capa $\mathrm{S}$ (capa superficial) en la superficie de Lactobacillus helveticus y Lactobacillus crispatus que pueden obstruir la adhesión de Escherichia coli O157: H7 (23).

La formación de biopelículas por parte de cepas probióticas también juega un papel importante en la inhibición de la adhesión de los microrganismos patógenos, ya que la formación de estas previene la colonización por parte de patógenos y desempeñan un papel importante como barrera protectora. Se ha visto que Lactobacillus casei Shirota y Lactobacillus rhamnosus forman biopelículas, inhibiendo así la adhesión de Listeria monocytogenes (24).

\section{Tratamiento de enfermedades en humanos Colonización del microbioma}

Las funciones de los probióticos están entrelazadas con los microrganismos que colonizan a los humanos. La comunicación cruzada entre los probióticos y las células huésped, o los probióticos y los microorganismos residentes, proporciona un medio clave para influir en la salud del huésped. El intestino contiene una gran cantidad de microorganismos, localizados principalmente en el colon, más de cuarenta billones de bacterias se albergan en el colon de un ser humano adulto. La interacción normal entre las bacterias intestinales y su huésped se conoce como una relación simbiótica. La presencia de un gran número de estructuras linfoides organizadas en la mucosa del intestino delgado (placas de Peyer) y del intestino grueso (folículos linfoides aislados) sugiere una importante influencia de las bacterias intestinales sobre la función inmunitaria. Muchos estudios han demostrado que las poblaciones de microrganismos colonizadores difieren entre individuos sanos y otros con enfermedades o condiciones insalubres. Ciertas bacterias comensales, como, Roseburia, Akkermansia, Bifidobacterium y Faecalibacterium prausnitzii parecen estar asociadas más comúnmente con la salud, pero es un área actualmente está en investigación, que busca determinar si la suplementación con estas bacterias puede mejorar la salud o revertir la enfermedad (25).

Por lo tanto, el uso beneficioso de la microflora intestinal, también conocida como "resistencia de colonización" o "efecto barrera" es un mecanismo importante utilizado por las bacterias intestinales autóctonas para mantener su presencia y conferir protección contra microorganismos recién ingeridos, incluidos los patógenos. Por consiguiente, la manipulación del microbioma intestinal por probióticos aumenta los números relativos de "bacterias beneficiosas", que tienen ciertos efectos sobre la función inmune, la digestión, el metabolismo y la comunicación entre el cerebro y el intestino. Los datos científicos demuestran que cepas probióticas específicas o combinaciones de cepas pueden ser beneficiosas en diferentes enfermedades. Los efectos pueden ser un efecto directo de la bacteria probiótica en sí, o un efecto indirecto a través de la interacción con el microbioma $(26,27)$.

\section{Aplicaciones clínicas}

El uso de probióticos para beneficios en la salud en humanos es un área prometedora en la actualidad. Una revisión de la literatura reveló que los probióticos se han probado en una amplia variedad de indicaciones (Figura 1). En 420 ensayos aleatorios controlados realizados entre los años 1977 a 2014, se encontró que sus indicaciones más comunes son, prevención de la diarrea asociada a 
antibióticos $(17 \%)$, tratamiento de la infección por Helicobacter pylori (16\%), tratamiento de la diarrea aguda pediátrica $(16 \%)$, prevención de alergias $(12 \%)$, tratamiento de la enfermedad crónica del intestino irritable $(10 \%)$ o enfermedad inflamatoria intestinal $(7 \%)$ y tratamiento de la vaginitis y la vaginosis bacteriana (6\%); con menos frecuencia, prevención de enterocolitis necrosante en recién nacidos $(3 \%)$, prevención de la diarrea del viajero $(3 \%)$, tratamiento de la diarrea aguda en adultos $(3 \%)$, tratamiento del estreñimiento (3\%) y tratamiento de la infección por Clostridium difficile $(3 \%)$; $y$, raramente, para sepsis, infecciones dentales y obesidad (1\% cada uno)(28). En la tabla 1 se describen estudios de las aplicaciones clínicas de diversos probióticos en la actualidad.

Algunas cepas probióticas son útiles para reducir la gravedad y la duración de la diarrea infecciosa aguda en niños, su administración oral acorta la duración de la enfermedad diarreica aguda en los niños en aproximadamente un día. En la prevención de la diarrea asociada a antibióticos existe una fuerte evidencia de su eficacia en adultos o niños que están recibiendo terapia con antibióticos (25).

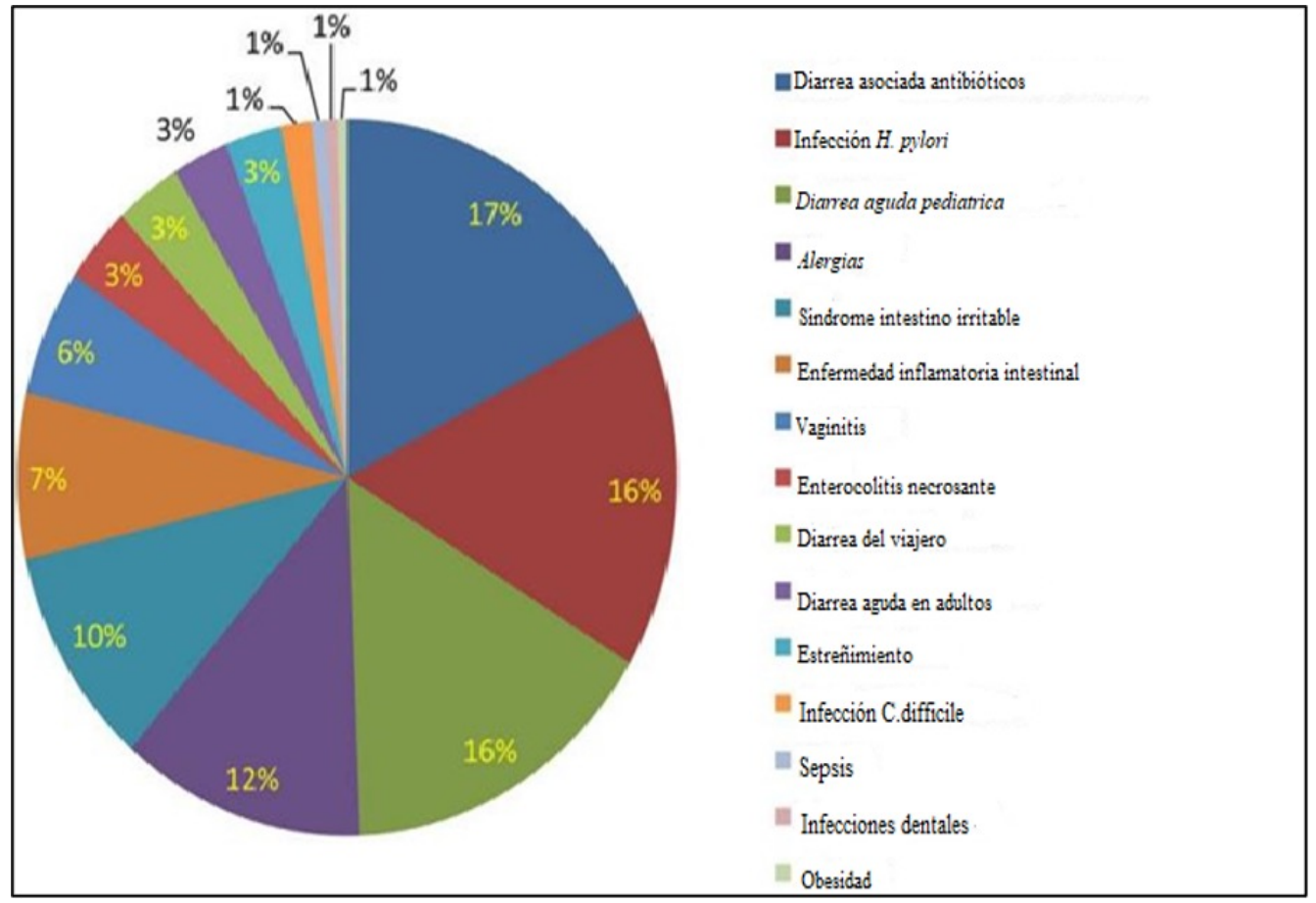

Figura 1. Indicaciones más comunes de los probióticos. Se representan las quince indicaciones más comúnmente estudiadas para los probióticos obtenidas a partir de 420 ensayos controlados aleatorios, 1977- 2014. Adaptado de (28).

Un metaanálisis realizado en el año 2016, concluyó que los probióticos pueden reducir el riesgo de desarrollar diarrea asociada a C. difficile (DAAC) en pacientes que reciben antibióticos. El uso de probióticos redujo significativamente el riesgo de desarrollar DAAC en un $60.5 \%$, los cuales demostraron ser beneficiosos tanto en adultos como en niños, especialmente entre pacientes hospitalizados. Lactobacillus, Saccharomyces y una mezcla de probióticos fueron todos beneficiosos para reducir el riesgo de desarrollar DAAC. Sin embargo, los autores advierten que se necesitan estudios adicionales para determinar la mejor dosificación (47).

Igualmente, para la infección de H.pylori se sugiere que la suplementación de antibióticos anti H. pylori con ciertos probióticos puede ser efectiva para aumentar las tasas de erradicación, y puede considerarse útil para los pacientes con fallas en dicha erradicación (33). 
Tabla 1. Evidencia de usos de probióticos en la salud humana

\begin{tabular}{|c|c|c|c|}
\hline & Especie probiótica & Conclusión del estudio & Referencia \\
\hline \multirow[t]{3}{*}{$\begin{array}{l}\text { Diarrea } \\
\text { asociada a } \\
\text { antibióticos } \\
\text { (DAA) }\end{array}$} & $\begin{array}{l}\text { Bifidobacterium spp., Bacillus spp., } \\
\text { Clostridium butyricum, Lactobacilli } \\
\text { spp., } \\
\text { Lactococcus spp, } \\
\text { Leuconostoc cremoris y } \\
\text { Streptococcus spp. }\end{array}$ & $\begin{array}{l}\text { Los probióticos son prometedores para la } \\
\text { prevención de la DAA pediátrica en niños (0- } \\
18 \text { años). }\end{array}$ & (29) 2018 \\
\hline & Saccharomyces boulardii & $\begin{array}{l}\text { Redujo el riesgo de diarrea asociada a } \\
\text { antibióticos en pacientes tratados con } \\
\text { antibióticos }\end{array}$ & (30) 2015 \\
\hline & Bacillus Licheniformis & $\begin{array}{l}\text { Efectivo en la prevención de } \mathrm{AAD} \text { en adultos } \\
\text { mayores. }(>65 \text { años })\end{array}$ & (31) 2015 \\
\hline \multirow{5}{*}{$\begin{array}{l}\text { Terapia para la } \\
\text { erradicación de } \\
\text { Helicobacter } \\
\text { pylori }\end{array}$} & Lactobacillus reuteri & Erradicación $H$. pylori altas. & (32) 2012 \\
\hline & Lactobacillus rhamnosus & Tasas de erradicación altas. & (33) 2014 \\
\hline & Saccharomyces boulardi & $\begin{array}{l}\text { Reduce los efectos adversos yaumenta } \\
\text { la tolerabilidad de los regímenes } \\
\text { de erradicación de H. pylori }\end{array}$ & (34) 2017 \\
\hline & $\begin{array}{l}\text { Bifidobacterium animalis subsp. } \\
\text { (DSM15954), } \\
\text { Lactobacillus rhamnosus } \mathrm{GG} \\
\text { lactis }\end{array}$ & $\begin{array}{l}\text { Reducción de los efectos secundarios } \\
\text { relacionados con la terapia. }\end{array}$ & (35) 2015 \\
\hline & Lactobacillus casei & Tasa de erradicación de H.pylori & (36) 2017 \\
\hline \multirow{2}{*}{$\begin{array}{l}\text { Prevención de } \\
\text { la diarrea } \\
\text { asociada a } \\
\text { Clostridium } \\
\text { difficile } \\
\text { (DAAC) } \\
\end{array}$} & $\begin{array}{l}\text { L. casei, L. bulgaricus, YS. } \\
\text { thermophilus }\end{array}$ & $\begin{array}{l}\text { Reduce la incidencia de diarrea asociada a } C \text {. } \\
\text { difficile }\end{array}$ & (37) 2012 \\
\hline & $\begin{array}{l}\text { Lactobacillus acidophilus } \\
\text { CL1285 y Lactobacillus casei } \\
\text { LBC80R }\end{array}$ & $\begin{array}{l}\text { Eficacia en la prevención de diarrea asociada a } \\
\text { C. difficile }\end{array}$ & (38) 2012 \\
\hline \multirow{5}{*}{$\begin{array}{l}\text { Síndrome del } \\
\text { intestino } \\
\text { irritable (SII) }\end{array}$} & $\begin{array}{l}\text { Bifidibacterium lactis } \\
\text { CNCM I-2494 }\end{array}$ & Acelera el tránsito gastrointestinal. & (39) 2018 \\
\hline & Bifidobacterium infantis 35624 & $\begin{array}{l}\text { Reduce el dolor / malestar abdominal, } \\
\text { hinchazón / distensión y dificultad para } \\
\text { defecar. }\end{array}$ & (39) 2018 \\
\hline & Faecalibacterium prausnitzii & $\begin{array}{l}\text { Induce IL-10 en células dendríticas humanas y } \\
\text { murinas y previene el desarrollo de inflamación } \\
\text { crónica. }\end{array}$ & (40) 2014 \\
\hline & L. casei Shirota & Mejora en el estreñimiento. & (41) 2014 \\
\hline & $\begin{array}{l}\text { Lactobacillus rhamnosus } \\
\text { NCIMB 30174, } \\
\text { L. plantarum } \\
\text { NCIMB 30173, } \\
\text { L. acidophilus } \\
\text { NCIMB 30175, y } \\
\text { Enterococcus faecium } \\
\text { NCIMB } 30176 .\end{array}$ & Reduce dolor intestinal y mejora el hábito & (41) 2014 \\
\hline $\begin{array}{l}\text { Mala digestión } \\
\text { de la lactosa }\end{array}$ & $\begin{array}{l}\text { Lactobacillus delbrueckii subsp. } \\
\text { Bulgaricus, y } \\
\text { Streptococcus thermophilus }\end{array}$ & $\begin{array}{l}\text { Reducción de los síntomas asociados a la mala } \\
\text { digestión de la lactosa. }\end{array}$ & (42) 2010 \\
\hline \multirow[t]{2}{*}{$\begin{array}{l}\text { Enterocolitis } \\
\text { necrotizante } \\
\text { (ECN) }\end{array}$} & $\begin{array}{l}\text { Lactobacillusacid-Ophilus y } \\
\text { Bifidobacterium bifidum. }\end{array}$ & $\begin{array}{l}\text { Menor riesgo de enterocolitis } \\
\text { necrotizante quirúrgica en niños. }\end{array}$ & (43) 2018 \\
\hline & $\begin{array}{l}\text { Lactobacillus acidophilu, } \\
\text { Bifidobacterium infantis }\end{array}$ & $\begin{array}{l}\text { Reducción de enterocolitis necrotizante en } \\
\text { neonatos. }\end{array}$ & (44) 2017 \\
\hline Vaginitis & $\begin{array}{l}\text { Lactobacillus acidophilus, } \\
\text { Lactobacillus fermentum, } \\
\text { Lactobacillus rhamnosus y } \\
\text { Lactobacillus gasseri }\end{array}$ & $\begin{array}{l}\text { Capaces de producir biosurfactantes, } \\
\text { confiriendo una acción probiótica contra } \\
\text { patógenos }\end{array}$ & $(45) 2014$ \\
\hline Obesidad & L. rhamnosus GGMCC. & Reducción de peso & $(46) 2016$ \\
\hline
\end{tabular}

\section{1}


Una reducción en la distensión abdominal y la flatulencia como resultado de los tratamientos probióticos es un hallazgo consistente en los estudios publicados; algunas cepas pueden aliviar el dolor y proporcionar alivio global. La literatura sugiere que ciertos probióticos pueden aliviar los síntomas producidos por SII y mejorar la calidad de vida en pacientes con dolor abdominal funcional (25). En una serie de estudios controlados con personas que consumen yogurt con cultivos vivos, se confirmó que Streptococcus thermophilus y Lactobacillus delbrueckii subsp. bulgaricus mejoran la digestión de la lactosa y reducen los síntomas relacionados con la intolerancia a esta (42).

El uso de probióticos también se ha estudiado como una terapia para disminuir el riesgo de enterocolitis necrosante (ECN), un trastorno asintomático que afecta principalmente a los recien nacidos prematuros (48). La administración de suplementos probióticos reduce el riesgo de $\mathrm{ECN}$ en recién nacidos prematuros. Los metaanálisis de ensayos aleatorios controlados también han mostrado un riesgo reducido de muerte en los grupos tratados con probióticos, aunque cabe resaltar que no todas las preparaciones probióticas probadas fueron efectivas (25).

Preparados de probióticos disponibles en el mercado Actualmente, los pacientes con trastornos gastrodigestivos se encuentran atraídos e interesados por tomar probióticos como un tratamiento complementario, al igual que las personas que presentan condiciones "normales" digestivas, como una medida preventiva. Por lo que la necesidad de crear productos que contengan estas cepas es cada vez alta. De acuerdo con estudios realizados y revisión de literatura, se han elaborado preparados diversos probióticos comerciales que se pueden encontrar en el mercado, ejemplos de ellos se muestran en la tabla 2.

Tabla 2. Ejemplos de diferentes tipos de productos probióticos encontrados en el mercado

\begin{tabular}{|c|c|c|c|}
\hline Cepa probiótica & Formulación & Nombre comercial & Evidencia basada en la eficacia \\
\hline $\begin{array}{l}\text { Bifidobacterium animalis subsp } \\
\text { lactis } \mathrm{DN}-173010\end{array}$ & Yogurt & Activia (Danone) & Estreñimiento \\
\hline Bifidobacterium infantis 35624 & Bebida, capsula & $\begin{array}{l}\text { Align (Procter \& } \\
\text { Gamble) }\end{array}$ & SII \\
\hline Clostridium butyricum 588 & Tabletas, bebida & $\begin{array}{l}\text { MIYA-BM } \\
\text { (Miyarisan Pharm) }\end{array}$ & $\begin{array}{l}\text { DAA } \\
\text { Infección por helicobacter pylori }\end{array}$ \\
\hline $\begin{array}{l}\text { Enterococcus faecium } \\
\text { SF } 68\end{array}$ & Polvo, Sachets & $\begin{array}{l}\text { Bioflorin(Cerbios- } \\
\text { Pharma) }\end{array}$ & Diarrea adulta aguda \\
\hline Lactobacillus acidophilus Lb & Capsulas, Sachets & $\begin{array}{l}\text { Lacteol (PUMC } \\
\text { Pharm) }\end{array}$ & Diarrea aguda en niños. \\
\hline $\begin{array}{l}\text { Lactobacillus casei } \\
\text { subsp Shirota }\end{array}$ & Leche fermentada & Yakult (Yakult) & $\begin{array}{l}\text { Estreñimiento Infección por } \\
\text { helicobacter pylori }\end{array}$ \\
\hline L. casei DN-114001 & $\begin{array}{l}\text { Leche fermentada, } \\
\text { yogurt }\end{array}$ & $\begin{array}{l}\text { Actimel, DanActive } \\
\text { (Danone) }\end{array}$ & $\begin{array}{l}\text { DAA, prevención de la diarrea } \\
\text { pediátrica, } \\
\text { infecciones respiratorias }\end{array}$ \\
\hline L. rhamnosus Lcr35 & Capsulas vaginales & Gynophilus & VB \\
\hline $\begin{array}{l}\text { Saccharomyces boulardii CNCM } \\
\text { I-745 (lyo) }\end{array}$ & Capsulas & $\begin{array}{l}\text { Florastor,Codex, } \\
\text { UltraLevure } \\
\text { (Biocodex) }\end{array}$ & $\begin{array}{l}\text { DAA, ICD, diarrea aguda en } \\
\text { adultos y pediátrica, DV, infección } \\
\text { por } H \text {. pylori }\end{array}$ \\
\hline $\begin{array}{l}\text { L. acidophilus CL1285 } \\
+ \text { L. casei Lbc80r + L.rhamnosus } \\
\text { CLR2 }\end{array}$ & $\begin{array}{l}\text { Bebidas fermentadas, } \\
\text { capsulas }\end{array}$ & Bio $\mathrm{K}+(\mathrm{BioK}+\mathrm{Intl})$ & DAA, SII \\
\hline $\begin{array}{l}\text { Lactobacillus } \\
\text { helveticusR0052(CNCM I-1722) }\end{array}$ & Capsulas, sachets & $\begin{array}{l}\text { Lacidofil } \\
\text { (Lallemand) }\end{array}$ & Infección por $H$. pylori, DAA \\
\hline
\end{tabular}


+ L.Rhamnosus R0011(CNCM I1720)

L.helveticus(bulgaricus) $4962+$

L. acidophilus

L. reuteri DSM17938 +L. reuteri PTA5289

Bacillus clausii (4 cepas: O/C, N/R84, T84, Sin8)

Bifidobacterium breve,

Bifidobacterium longum, $B$. infantis,

L. acidophilus,

L. plantarum, L. casei,

L. bulgaricus, Streptococcu thermophilus

Abreviaturas: DAA, diarrea asociada a antibióticos; VB, vaginosis bacteriana; ICD, infección por Clostridium difficile; SII, síndrome del intestino irritable; DV, diarrea del viajero; CU colitis ulcerosa. Tomado de (28).
A’Biotica (Institut

Rosell)

Lactinex (BD Diarrea aguda en adultos.

Diagnostics)

Prodentis (BioGaia) Infecciones dentales

cápsulas

VSL\#3 (Sigma-Tau SII CU

Pharm Inc)
Cápsulas, esporas en

vial

Enterogermina

(SanofiAventis)

Antidiarreico

Sachets
Para que los cultivos probióticos sean efectivos se deben tener en cuenta ciertas condiciones como la estabilidad, viabilidad y la dosis que se suministra. La estabilidad hace referencia a que deben ser capaces de resistir las condiciones de procesamiento, conservar sus propiedades probióticas después del procesamiento y sobrevivir en cantidades suficientes en el producto durante su almacenamiento. La estabilidad de un probiótico está vinculada a diversos factores, incluidos el género, la especie, el biotipo de la cepa y, sobre todo, las condiciones de almacenamiento de la formulación. Al igual que la estabilidad, la viabilidad también depende mucho de la especie, especialmente de las características farmacocinéticas de estas cuando son suministradas en el tracto gastrointestinal. Aunque existen situaciones donde la variabilidad no es un factor importante, como por ejemplo cuando son usados para mejorar la digestión de la lactosa, con efectos antihipertensivos $\mathrm{y}$ en algunas actividades de modulación del sistema inmune. Ciertos efectos se han relacionado con células no viables como componentes celulares, actividades enzimáticas o productos de fermentación. Por último, los efectos probióticos parecen ser dependientes de la dosis. Sin embargo, el efecto de la dosis es controversial, aunque la dosis habitual efectiva que se tiene en humano se encuentra en un rango de 107-9 UFC/mg por día (49).

\section{CONCLUSIONES}

Los probióticos son una novedosa alternativa en el tratamiento contra patógenos y en la prevención de algunas enfermedades en humanos, lo que se corrobora con los diferentes estudios que se obtuvieron en esta revisión en los cuales se observó el beneficio de varias cepas cuando fueron evaluadas en humanos que presentaban alguna enfermedad. Demostrando que el uso de diferentes cepas con actividad probiótica contribuye en la reducción del agente causal de la enfermedad, reducción de los síntomas, inducción de la actividad inmune o disminución en el riesgo de contraer la infección.

El uso de antibióticos para el tratamiento contra patógenos puede generar efectos adversos lo que ha llevado al estudio de los probióticos dadas sus propiedades para contrarrestar este tipo de infecciones sin generar un efecto negativo. En esta revisión se pudo establecer la actividad probiótica de diferentes cepas de las cuales se destacan los géneros de Lactobacillus, Bacillus, Enterococcus y Lactococcus, los cuales mostraron actividades antimicrobianas in-vitro e in-vivo.

Se recomienda que a la hora de usar probióticos se tengan en cuenta la descripción detallada del probiótico: género, especie, cepa, dosis, duración e 
indicaciones, dado que muchas de sus propiedades son específicas de las cepas, los patógenos o de su uso indicado.

CONFLICTOS DE INTERESES: Los autores no declaran conflictos de interés.

\section{REFERENCIAS}

1. FAO/WHO (Food and Agriculture Organization of the United Nations, World Health Organization). Probiotics in food. Health and nutritional properties and guidelines for evaluation. Food and agriculture organization of the United Nations and World Health Organization, Rome. FAO food and nutrition paper no. 85.2002

2. Oyetayo, V. O., \& Oyetayo, F. L. Potential of probiotics as biotherapeutic agents targeting the innate immune system. African Journal of Biotechnology. $2005 ; 4,123-127$.

3. Shah, N. Functional cultures and health benefits. International Dairy Journal. 2007; 17(11), 1262-1277.

4. Kollath, W. Nutrition and the tooth system; general review with special referenceto vitamins. Deutsche zahnarztliche Zeitschrift.1953; 8, 7-16.

5. Hamilton-Miller, J., Gibson, G., \& Bruck, W. Some insights into the derivation and early uses of the word 'probiotic'. British Journal Of Nutrition. 2003; 90(04), 845.

6. Reid, G. Probiotics: definition, scope and mechanisms of action. Best Practice \& Research Clinical Gastroenterology. 2016; 30(1), 17-25.

7. Hill, C., Guarner, F., Reid, G., Gibson, G., Merenstein, D., \& Pot, B. The International Scientific Association for Probiotics and Prebiotics consensus statement on the scope and appropriate use of the term probiotic. Nature Reviews Gastroenterology \& Hepatology. 2014; 11(8), 506-514.

8. Amara, A., \& Shibl, A. Role of Probiotics in health improvement, infection control and disease treatment and management. Saudi Pharmaceutical Journal. 2015; 23(2), 107-114.

9. Fuller, R. Probiotics in man and animals. Journal of Applied Bacteriology. 1989 66,365-378.
10. Oelschlaeger, Tobias A. 2010. "Mechanisms Of Probiotic Actions - A Review". International Journal Of Medical Microbiology.2010; 300 (1), 57-62.

11. Wan, L. Y. M., Chen, Z. J., Shah, N. P., \& ElNezami, H. (2016). Modulation of intestinal epithelial defense responses by probiotic bacteria. Critical Reviews in Food Science and Nutrition.2016;56, 2628 2641.

12. BalcÃ $j z a r, ~ J o s \tilde{~ L u i s, ~ I g n a c i o ~ d e ~ B l a s, ~ I m a n o l ~}$ Ruiz-Zarzuela, Daniel Vendrell, Olivia Giron $\tilde{A} \odot s$, and Jos Ã Luis Muzquiz. "Enhancement Of The Immune Response And Protection Induced By Probiotic Lactic Acid Bacteria Against Furunculosis In Rainbow Trout (Oncorhynchus Mykiss)". FEMS Immunology \& Medical Microbiology.2007; 51 (1): 185-193.

13. Cleusix, Valentine, Christophe Lacroix, Sabine Vollenweider, and GwenaÃ«lle Le Blay. "Glycerol Induces Reuterin Production And Decreases Escherichia Coli Population In An In Vitro Model Of Colonic Fermentation With Immobilized Human Feces". FEMS Microbiology Ecology.2008; 63 (1): 5664.

14. Dasari S, Anandan S, Rajendra W, Valluru L. Role of microbial flora in female genital tract: A comprehensive review. Asian Pacific Journal of Tropical Disease. 2016;6(11):909- 917.

15. Corr, S. C., Y. Li, C. U. Riedel, P. W. O'Toole, C. Hill, and C. G. M. Gahan. "Bacteriocin Production As A Mechanism For The Antiinfective Activity Of Lactobacillus Salivarius UCC118". Proceedings Of The National Academy Of Sciences.2007; 104 (18): 7617-7621.

16. Servin, Alain L. "Antagonistic Activities Of Lactobacilli And Bifidobacteria Against Microbial Pathogens". FEMS Microbiology Reviews. 2004; 28 (4): 405-440.

17. Alvarez-Olmos, M. I., and R. A. Oberhelman. "Probiotic Agents And Infectious Diseases: A Modern Perspective On A Traditional Therapy". Clinical Infectious Diseases.2001; 32 (11)

18. Asahara, T., K. Shimizu, K. Nomoto, T. Hamabata, A. Ozawa, and Y. Takeda. "Probiotic Bifidobacteria Protect Mice From Lethal Infection With Shiga Toxin-Producing Escherichia Coli 
O157:H7". Infection And Immunity. 2004; 72 (4): 2240-2247.

19. Chaucheyras-Durand, F., N.D. Walker, and A. Bach. "Effects Of Active Dry Yeasts On The Rumen Microbial Ecosystem: Past, Present And Future". Animal Feed Science And Technology. 2008;145 (14): 5-26.

20. Kim, Younghoon, Sejong oh, and Sae Hun Kim. "Released Exopolysaccharide (R-EPS) Produced From Probiotic Bacteria Reduce Biofilm Formation Of Enterohemorrhagic Escherichia Coli O157:H7". Biochemical And Biophysical Research Communications.2009; 379 (2): 324-329.

21. Liu, Zhengqi, Zhihong Zhang, Liang Qiu, Fen Zhang, Xiongpeng Xu, Hua Wei, and Xueying Tao. "Characterization And Bioactivities Of The Exopolysaccharide From A Probiotic Strain Of Lactobacillus Plantarum WLPL04". Journal Of Dairy Science. 2017;100 (9): 6895-6905.

22. La Ragione, R.M., A. Narbad, M.J. Gasson, and M.J. Woodward. "In Vivo Characterization Of Lactobacillus Johnsonii FI9785 For Use As A Defined Competitive Exclusion Agent Against Bacterial Pathogens In Poultry". Letters In Applied Microbiology.2004;38 13): 197-205. doi:10.1111/j.1472-765x.2004.01474.x

23. Johnson-Henry, Kathene C., Karen E. Hagen, Mahsa Gordonpour, Thomas A. Tompkins, and Philip M. Sherman. "Surface-Layer Protein Extracts From Lactobacillus Helveticus Inhibit Enterohaemorrhagic Escherichia Coli O157:H7 Adhesion To Epithelial Cells". Cellular Microbiology.2007; 9 (2): 356-367.

24. UNAL TURHAN, Emel, Zerrin ERGINKAYA, Melek Hatice UNEY, and Emir Ayse OZER. "Listeria Monocytogenes'In Gelişimi Üzerine Probiyotik Biyofilmlerin İnaktivasyon Etkisi". Kafkas Universitesi Veteriner Fakultesi Dergisi. 2017.

25. Guarner F, Khan AG, Garisch J, et al. World Gastroenterology Organisation Global Guidelines: probiotics and prebiotics October 2011. J Clin Gastroenterol 2012;46:468-81.

26. George Kerry, R., Patra, J., Gouda, S., Park, Y., Shin, H., \& Das, G. Benefaction of probiotics for human health: A review. Journal Of Food And Drug Analysis. 2018.
27. Scott, K., Antoine, J., Midtvedt, T., \& van Hemert, S.. Manipulating thegut microbiota to maintain health and treat disease. Microbial Ecology In Health \& Disease. $2015 ; 26(0)$.

28. McFarland, L. From Yaks to Yogurt: The History, Development, and Current Use of Probiotics. Clinical Infectious Diseases.2015; 60(suppl 2), S85S90.

29. Hayes, Shelby R., and Ashley J. Vargas. "Probiotics For The Prevention Of Pediatric AntibioticAssociated Diarrhea".2018.

30. Szajewska, H., \& Kołodziej, M. Systematic review with meta-analysis:Saccharomyces boulardiiin the prevention of antibiotic-associated diarrhoea. Alimentary Pharmacology \& Therapeutics.2015; 42(7), 793-801

31. Xie, C., Li, J., Wang, K., Li, Q., \& Chen, D. Probiotics for the prevention of antibiotic- associated diarrhoea in older patients: A systematic review. Travel Medicine And Infectious Disease.2015;13(2), 128-134.

32. Ojetti, V., Bruno, G., Ainora, M., Gigante, G., Rizzo, G., Roccarina, D., \& Gasbarrini, A. Impact of Lactobacillus reuteri Supplementation on AntiHelicobacter pylori Levofloxacin- Based Second-Line Therapy. Gastroenterology Research And Practice, 2012, 1-6.

33. Dang, Y., Reinhardt, J., Zhou, X., \& Zhang, G. The Effect of Probiotics Supplementation on Helicobacter pylori Eradication Rates and Side Effects during Eradication Therapy: A Meta-Analysis. Plos ONE.2014; 9(11), e111030.

34. Wang, Z., Chen, X., Zhang, Z., Li, Y., Deng, J., $\& \mathrm{Tu}, \mathrm{J}$. et al. Effects of anti- Helicobacter pylori concomitant therapy and probiotic supplementation on the throat and gut microbiota in humans. Microbial Pathogenesis.2017: 109, 156-161.

35. Hauser G, Salkic N, Vukelic K, JajacKnez A, Stimac D. Probiotics for standard triple Helicobacter pylori eradication: a randomized, double-blind, placebo-controlled trial. Medicine (Baltimore). 2015 May;94(17):e685.

36. Feng, J., Wang, F., Qiu, X., McFarland, L., Chen, P., \& Zhou, R. et al. Efficacy and safety of 
probiotic-supplemented triple therapy for eradication of Helicobacter pylori in children: a systematic review and network meta-analysis. European Journal of Clinical Pharmacology,.2017;73(10), 1199-1208.

37. Friedman, G. The Role of Probiotics in the Prevention and Treatment of Antibiotic- Associated Diarrhea and Clostridium Difficile Colitis. Gastroenterology Clinics Of North America.2012; 41(4), 763-779.

38. Johnson, S., Maziade, P., McFarland, L., Trick, W., Donskey, C., \& Currie, B. et al. Is primary prevention of Clostridium difficile infection possible with specific probiotics?. International Journal Of Infectious Diseases.2012; 16(11), e786-e792.

39. Principi, N., Cozzali, R., Farinelli, E., Brusaferro, A., \& Esposito, S. Gut dysbiosis and irritable bowel syndrome: The potential role of probiotics. Journal Of Infection.2018; 76(2), 111-120.

40. Cao Y, Shen J, Ran ZH. Association between Faecalibacterium prausnitzii reduction and inflammatory bowel disease: a meta-analysis and systematic review of the literature. Gastroenterol Res Pract.2014;872725.

41. Ford, A., Quigley, E., Lacy, B., Lembo, A., Saito, Y., \& Schiller, L. et al. Efficacy of Prebiotics, Probiotics and Synbiotics in Irritable Bowel Syndrome and Chronic Idiopathic Constipation: Systematic Review and Meta-analysis. The American Journal Of Gastroenterology.2014; 109(10), 1547-1561.

42. EFSA Panel on Dietetic Products $\mathrm{N}$ and $\mathrm{A}$ (NDA). Scientific Opinion on the substantiation of health claims related to live yoghurt cultures and improved lactose digestion (ID 1143,2976) pursuant to Article 13(1) of Regulation (EC) No 1924/2006. EFSA J. 2010 Oct 1;8(10): n/a-n/a.

43. Patel, R., \& Underwood, M. Probiotics and necrotizing enterocolitis. Seminars In Pediatric Surgery.2018; 27(1), 39-46.

44. Underwood, M. Impact of probiotics on necrotizing enterocolitis. Seminars In Perinatology.2017;41(1), 41-51.

45. Cancelo Hidalgo, M., Neyro Bilbao, J., \& Baquero Úbeda, J. Tratamiento adyuvante de la vaginitis con probióticos. Grado de acuerdo basado en el método Delphi. Progresos De Obstetricia Y Ginecología.2014; 57(1), 4-13.

46. Rouxinol-Dias, A., Pinto, A., Janeiro, C., Rodrigues, D., Moreira, M., Dias, J., \&Pereira, P. Probiotics for the control of obesity - Its effect on weight change. Porto Biomedical Journal.2016;1(1), 12-24.

47. Chamberlain, R., \& Lau, C. Probiotics are effective at preventing Clostridium difficile- associated diarrhea: a systematic review and meta-analysis. International Journal Of General Medicine.2016; 27. 48. Patel, R., \& Underwood, M. Probiotics and necrotizing enterocolitis. Seminars In Pediatric Surgery.2018; 27(1), 39-46.

49. Minelli, E., \& Benini, A. Relationship between number of bacteria and their probiotic effects. Microbial Ecology In Health \& Disease.2008; 20(4). 